\title{
Gastrointestinal uptake of liposomes. I. In vitro and in situ studies
}

\author{
Chia-Ming Chiang and Norman Weiner \\ College of Pharmacy, The University of Michigan, Ann Arbor, MI 48109 (U.S.A.)
}

(Received 27 October 1986)

(Revised version received 15 December 1986)

(Accepted 16 December 1986)

\begin{abstract}
Key words: Liposomal entrapment; Oral drug delivery; Everted sac, Doluisio method; Difussion cell
\end{abstract}

\section{Summary}

The stability of L- $\alpha$-phosphatidylcholine distearoyl/cholesterol and egg lecithin/cholesterol multilamellar liposomes at acidic $\mathrm{pH}$ values, in the presence of bile salts and in the presence of pancreatic lipase, was studied by determining the leakage rates of entrapped markers. Substantial loss of entrapped glucose and complete loss of entrapped carboxyfluorescein were observed under conditions similar to what would be expected in the gastrointestinal tract. Transport studies using in vitro diffusion cells and everted-sac experiments suggest that intact liposomes are not taken up by epithelial cells and that liposomal entrapment does not facilitate the transport of non-absorbable drugs and prevents the transport of absorbable ones. In situ studies seem to confirm this viewpoint since almost all of the liposomes and their entrapped markers remain in the intestine even after $3 \mathrm{~h}$.

\section{Introduction}

Attempts to use liposomes as an oral dosage form have been a major focus in drug delivery research since it has been suggested that liposomes may either protect their entrapped drugs from digestive degradation (Bangham et al., 1965) or increase the absorption of poorly absorbed drugs from the gastrointestinal tract (Sessa and Weissman, 1970). It has been suggested that intact liposomes which contain drugs can be endocytosed by the absorptive (Rowland and Woodley, 1981) cells of the gastrointestinal tract, thereby

Correspondence: C.-M. Chiang, College of Pharmacy, The University of Michigan, Ann Arbor, MI 48109, U.S.A. facilitating transport of molecules which could not traverse membranes in their free state.

Attempts have been made to increase the gastrointestinal absorption of water-soluble drugs (Ueno et al., 1982), water-insoluble drugs (Nagata et al., 1984), ionophores (Young et al., 1979), and a variety of polypeptides and proteins (Hemker, et al., 1980; Kawada, et al., 1981). Generally, these drugs have poor bioavailability when administered by the oral route using traditional dosage forms because they are labile in the gastrointestinal tract or have little intrinsic bioavailability. The results of these studies, with few exceptions (Nagata et al., 1984), indicate that liposomal entrapment either does not facilitate oral absorption (Young et al., 1979; Kawada et al., 1981) or its influences on absorption are not predictable and reproduci- 
ble (Arrieta-Molero et al., 1982; Tragl et al., 1979). Thus, despite 20 years of research in this area, the utility of liposomes as an oral drug delivery system is still open to question.

The present work is an investigation of factors with potential controlling influence on liposomal drug delivery by the oral route. With the aid of in vitro and in situ model systems, we have isolated and studied a number of parameters which have been reported to affect the uptake and disposition of drugs entrapped in multilamellar vesicles. In particular, we have sought insight into the following questions:

1. Do liposomes maintain their integrity in the physiological environment of the gastrointestinal tract?

2. Are non-absorbable markers (e.g., PEG-4000), when encapsulated into liposomes, able to traverse the gastrointestinal tract?

3. Does encapsulation into liposomes affect the gastrointestinal uptake of absorbable markers (e.g., glucose)?

\section{Materials and Methods}

\section{Materials}

The lipids used were $\mathrm{L}$ - $\alpha$-phosphatidylcholine distearoyl (Sigma Chemical Co., St. Louis, MO), egg phosphatidylcholine (Sigma) and cholesterol (Avanti Polar Lipids, Birmingham, AL). L- $\alpha$-Phosphatidylcholine distearoyl and cgg lecithin were used as purchased and stored below $0^{\circ} \mathrm{C}$. Cholesterol was recrystallized from ethanol solution 3 times. All liposome preparations that employed cholesterol were prepared using a phospholipid: cholesterol molar ratio of $2: 1$. Carboxyfluorescein (Eastman Kodak, Rochester, NY) was purified by the method described by Sila et al. (1986). All other chemicals were of reagent grade and used as obtained.

The following radiolabelled compounds were used: $\quad$ L- $\alpha$-phosphatidylcholine $\operatorname{di}\left[1-{ }^{14} \mathrm{C}\right]$ stearoyl (Amersham, Arlington Heights, IL) and $[1,2(n)$ $\left.{ }^{3} \mathrm{H}\right] \mathrm{cholesterol}$ (New England Nuclear, Boston, MA) were used as lipid markers and [1,2${ }_{14}$ C]PEG-4000 (Amersham), D- $\left[1,2,3,4,5,6-{ }^{14} \mathrm{C}\right]-$ glucose (Eastman Kodak), $\left[{ }^{14} \mathrm{C}\right]$ sucrose (ICN Ra- diochemicals, Irvine, CA), $\left[1,2-{ }^{3} \mathrm{H}\right]$ hydrocortisone (Amersham) and $\left[1,2-{ }^{3} \mathrm{H}\right]$ progesterone (Amersham) were used as entrapped markers. $\left[{ }^{3} \mathrm{H}\right]$ methanol (Amersham), $\left[1-{ }^{14} \mathrm{C}\right]$ ethanol (Amersham) and $\left[1-{ }^{14} \mathrm{C}\right]$ butanol (Amersham) were used as markers to validate the in vitro diffusion cell as a suitable model membrane system.

\section{Preparation of large multilammellar vesicles}

The lipid mixtures ( $32 \mu \mathrm{mol}$ phospholipid and $16 \mu \mathrm{mol}$ cholesterol) were dried from a chloroform solution at room temperature in a $20-\mathrm{ml}$ glass scintillation vial (Kimble, Toledo, $\mathrm{OH}$ ). Lipophilic compounds which were to be entrapped were dissolved together with the phospholipids in chloroform. A thin film was formed on the sides of the vial by hand agitation during the drying process and residual solvent was removed by storing the vial overnight under vacuum. The dried lipids were then hydrated with $1.0 \mathrm{ml}$ of $67 \mathrm{mM}$ phosphate buffer solution $(\mathrm{pH} \mathrm{7.0)}$ at a temperature above the transition temperature of the constituent phospholipid $\left(61^{\circ} \mathrm{C}\right.$ for liposomes composed of $\mathrm{L}$ - $\alpha$-phosphatidylcholine distearoyl/ cholesterol and room temperature for liposomes composed of egg lecithin/cholesterol). Watersoluble compounds which were to be entrapped were dissolved in buffer prior to the hydration step. Incubation of the lipid film with buffer was carried out for $30 \mathrm{~min}$ with intermittent vortexing. The suspension was then sonicated in a bath-type sonicator (Branson Cleaning Equipment Co., Shelton, $\mathrm{CN}$ ) for $30 \mathrm{~s}$ at the same temperature at which the films were hydrated, and the resultant dispersion was allowed to equilibrate for at least 4 $h$ at room temperature. The dispersion was passed through a $2.0-\mu \mathrm{m}$ polycarbonate filter (Nucleopore Corp., Pleasanton, CA) so as to remove large aggregates. The diameters of the resultant liposomes ranged from 0.2 to $1.3 \mu \mathrm{m}$ as determined by scanning transmission electron microscopy using a JEOL $100 \mathrm{CX}$ electron microscope (Jeol Ltd., Tokyo).

The suspension was then centrifuged 3 times at $10,000 \mathrm{rpm}$ for $5 \mathrm{~min}$ to remove untrapped marker from the liposome preparations. After each centrifugation step, the supernatant was removed and the liposomal pellet was resuspended in $2 \mathrm{ml}$ 
of fresh buffer. After the final centrifugation step, the supernatant was assayed to ensure complete removal of free marker.

Effect of acidity, bile salt and lipase on stability of liposomes

The stability of liposomes exposed to conditions that simulate the gastrointestinal tract was studied by determining the leakage rates of entrapped markers from liposomes at acidic $\mathrm{pH}$ values, in the presence of bile salts and in the presence of pancreatic lipase. L- $\alpha$-Phosphatidylcholine distearoyl/cholesterol or egg lecithin/ cholesterol liposomes containing either entrapped glucose $(0.3 \mathrm{M})$ with $\left[{ }^{14} \mathrm{C}\right]$ glucose or entrapped carboxyfluorescein as markers were prepared and dispersed in $10 \mathrm{mM}$ Tris buffer adjusted to $\mathrm{pH} 7.0$ with hydrochloric acid made isotonic with sodium chloride.

One-half $\mathrm{ml}$ of the glucose containing liposomes $(12 \mu \mathrm{mol})$ was incubated at $37^{\circ} \mathrm{C}$ for $2 \mathrm{~h}$ under the following conditions: (1) with $5.5 \mathrm{ml}$ buffer adjusted to a $\mathrm{pH}$ value of 2; (2) with $5.5 \mathrm{ml}$ buffer containing $10 \mathrm{mM}$ taurocholic acid (Mann Research Labs., Becton Dickenson, and Co., Rutherford, NJ); (3) with $3.5 \mathrm{ml}$ buffer containing pancreatic lipase $(10,000 \mathrm{U} / \mu \mathrm{mol}$ lipid) (Sigma) and $1 \mathrm{mM} \mathrm{CaCl}_{2}$. The $\mathrm{CaCl}_{2}$ is a lipase cofactor needed to maintain the activity of the pancreatic lipase. $0.5 \mathrm{ml}$ of the carboxyfluorescein containing liposomes $(12 \mu \mathrm{mol})$ was incubated at $37^{\circ} \mathrm{C}$ for 2 $h$ under conditions (2) and (3) as well as with a mixture of $10 \mathrm{mM}$ taurocholic acid and pancreatic lipase.

Leakage of glucose was determined by taking $200 \mu 1$ samples from the various incubation media at the start of the experiment (zero time) and every half hour thereafter for $2 \mathrm{~h}$. The samples were placed in an Amicon membrane cone (Amicon Corp., Danvers, MA) and $200 \mu 1$ Tris buffer was added. The membrane cone was fitted into a plastic support and the assembly was placed over a polycarbonate tube where the supernatant was collected upon centrifugation at $10,000 \mathrm{rpm}$ for $5 \mathrm{~min}$. $500 \mu \mathrm{l}$ of buffer was used to rinse the cone free of glucose which might be adsorbed on the cone surface. This washing procedure was repeated a second time and the total volume of supernatant (including washings) was measured and an aliquot was transferred into a vial containing $10 \mathrm{ml}$ scintillation fluid (Aquasol; New England Nuclear, Boston, MA) for counting in a scintillation counter (LS-9000; Beckman Instruments, Inc., Irvine, CA). An aliquot of the pellet was counted to determine the amount of glucose trapped at time zero.

Leakage of carboxyfluorescein as a function of time was determined by the method described by Sila et al. (1986). Briefly, fluorescence measurements (Model LS-5 spectrofluorometer, PerkinElmer) were performed using carboxylfluorescein concentrations $(\approx 100 \mathrm{mM})$ where the entrapped dye is completely self-quenched and little if any self-quenching is observed when carboxyfluorescein is released into the external medium. The liposomes were lysed by incubation with $1 \%$ Triton $\mathrm{X}-100$ for 15 minutes at $72^{\circ} \mathrm{C}$ to determine the amount of carboxylfluorescein trapped at time zero.

\section{In vitro uptake studies}

\section{Diffusion cell experiments}

The diffusion apparatus (Crown Glass Co., Somerville, NJ) which consists of two symmetrical cylindrical half-cells with a circular opening having a diameter of about $0.9 \mathrm{~cm}$ is the same as that described by Huq et al. (1986). Each chamber is fitted with a glass-stoppered port on top for addition or removal of material. The medium within the chambers was stirred by externally driven magnetic stirring bars placed in the circular depressions of the cell. The system was kept at $37^{\circ} \mathrm{C}$ by circulating water through the water jacket of the diffusion cell.

Male Sprague-Dawley rats weighing 300-350 g were anesthetized with $20 \%$ ethyl carbamate (Sigma) solution by two 1-ml i.p. injections $15 \mathrm{~min}$ apart. A jejunal segment was cut along the mesenteric border and rinsed with normal saline solution. A small piece of flattened intestinal membrane, slightly larger than the opening of the diffusion chamber, was cut and mounted between the half-cells with the mucosal side facing the donor compartment and the serosal side facing the receiver compartment. The rat was then sacrificed 
by ethyl carbamate injection into the heart.

Control experiments to test the suitability of the diffusion cell as a model system were run using radiolabelled alkanols (methanol, ethanol and butanol) dissolved in phosphate buffer. $\left[{ }^{3} \mathrm{H}\right]$ hydrocortisone or $\left[{ }^{3} \mathrm{H}\right]$ progesterone diluted with saturated solutions of their unlabelled counterparts served as free marker solutions for the control experiments. The liposomcs uscd as permeants in these studies were: (1) empty $\left[{ }^{14} \mathrm{C}\right] \mathrm{L}-\alpha-$ phosphatidylcholine distearoyl/cholesterol liposomes and (2) L- $\alpha$-phosphatidylcholine distearoyl/ cholesterol liposomes containing entrapped $\left[{ }^{3} \mathrm{H}\right]$ progesterone or $\left[{ }^{3} \mathrm{H}\right]$ hydrocortisone.

At the start of the experiment, the receiver compartment was filled with $1.8 \mathrm{ml}$ buffer and the donor compartment was filled with $1.8 \mathrm{ml}$ of either a buffered solution of free permeant or a liposomal dispersion (equilibrated to $37^{\circ} \mathrm{C}$ ). Samples were taken from the donor and receiver compartments at various time intervals for $2 \mathrm{~h}$ and the samples were assayed for radioactivity. The data for the permeation studies were plotted as total $\mathrm{cpm}$ in the receiver compartment as a function of time. Correction was made for dilution due to replacement of buffer after each sampling.

\section{Intestinal everted-sac experiments}

A modification of the everted-intestinal-sac technique described by Wilson and Weissman (1954) was used to study the transport of liposomally entrapped and free markers. Male SpragueDawley rats weighing $300-350 \mathrm{~g}$ were anesthetized with $20 \%$ ethyl carbamate solution by two $1-\mathrm{ml}$ i.p. injections $15 \mathrm{~min}$ apart. A $20-\mathrm{cm}$ segment of jejunum was quickly removed, rinsed with normal saline and everted. This segment was tied at one end with a linen ligature (Ethicon Inc., Somerville, $\mathrm{NJ}$ ), filled with oxygenated Krebs buffer and then tied at the other end. The resultant large sac was divided into $3-4-\mathrm{cm}$ sacs by tying at intervals. Each sac contained about $0.5 \mathrm{ml}$ pregassed Krebs buffer. The rat was then sacrificed by injection of ethyl carbamate into the heart.

L- $\alpha$-Phosphatidylcholine distearoyl/cholesterol liposomes containing an entrapped water-soluble marker, $\left[{ }^{3} \mathrm{H}\right] \mathrm{PEG}-4000$ or $\left[{ }^{14} \mathrm{C}\right]$ glucose, were used in these transport studies. In some experiments, double-labelled liposomes $\left(\left[{ }^{14} \mathrm{C}\right] \mathrm{L}-\alpha\right.$-phosphatidylcholine distearoyl $/\left[{ }^{3} \mathrm{H}\right] \mathrm{PEG}-4000$ or $\left[{ }^{3} \mathrm{H}\right]$ cholesterol $/\left[{ }^{14} \mathrm{C}\right]$ glucose) were used. The free marker solutions were prepared by diluting trace amounts of radiolabelled glucose or PEG-4000 with buffer.

Each small sac was individually placed in a $20-\mathrm{ml}$ glass vial containing $3 \mathrm{ml}$ oxygenated Krebs buffer kept in a $37^{\circ} \mathrm{C}$ water bath. After equilibration at this temperature for $5 \mathrm{~min}, 1 \mathrm{ml}$ of liposomal suspension was added to each vial to initiate the experiment. $100-\mu 1$ samples of the outside medium were taken every $30 \mathrm{~min}$ and replaced with buffer. At the end of the 2-h experiment, the everted sacs were removed from the vial, blotted dry and drained of the aqueous contents. Upon measuring the volume of the drained liquid, a $100-\mu 1$ aliquot was withdrawn and assayed by scintillation counting.

\section{In situ uptake studies}

The modified Doluisio technique that was used in these experiments was similar to that described by Schwinke et al. (1984). Briefly, a male Sprague-Dawley rat, weighing $250-300 \mathrm{~g}$, was fasted overnight and anesthetized with $20 \%$ ethyl carbamate solution by two 1-ml i.p. injections, 15 min apart. A midline abdominal incision was made and the small intestine was exposed. The proximal end of the jejunum (the $25-\mathrm{cm}$ segment of the intestine beginning at the distal end of the duodenum) was cannulated with an L-shaped glass tubing ( $4 \mathrm{~mm}$ o.d., $2 \mathrm{~mm}$ i.d.). A second cannula was then placed $22 \mathrm{~cm}$ distal to the first and the segment was rinsed with normal saline at $37^{\circ} \mathrm{C}$ until the washing appeared clear. This intestinal segment was arranged in S-shaped curves free of any constriction, covered with gauze, and kept moist with normal saline at $37^{\circ} \mathrm{C}$. The cannulae were then connected to the barrels of two plastic, disposable 5-ml syringes (Becton, Dickenson, \& Co., Rutherford, NJ) which were connected to a two-directional piston pump (Fluid Meter Inc., Oyster Bay, NJ). A final rinse of buffer solution was applied to the segment before the start of the experiment.

Empty L- $\alpha$-phosphatidylcholine distearoyl/ cholesterol liposomes containing a single lipid label $\left(\left[{ }^{14} \mathrm{C}\right] \mathrm{t}-\alpha\right.$-phosphatidylcholine distearoyl) or dual 
lipid labels $\left(\left[{ }^{14} \mathrm{C}\right] \mathrm{L}-\alpha\right.$-phosphatidylcholine distearoyl $/\left[{ }^{3} \mathrm{H}\right]$ cholesterol) were used. $\left[{ }^{14} \mathrm{C}[\mathrm{L}-\alpha\right.$-phosphatidylcholine distearoyl/cholesterol liposomes containing $\left[{ }^{3} \mathrm{H}\right] \mathrm{PEG}$ and $\mathrm{L}-\boldsymbol{\alpha}$-phosphatidylcholine distearoyl $/\left[{ }^{3} \mathrm{H}\right]$ cholesterol liposomes containing $\left[{ }^{14} \mathrm{C}\right]$ sucrose were also used. Free $\left[{ }^{14} \mathrm{C}\right]$ butanol dissolved in PBS in the presence of $\mathbf{L}-\alpha$-phosphatidylcholine distearoyl $/\left[{ }^{3} \mathrm{H}\right]$ cholesterol liposomes served as a control.

Initially, attempts were made to determine the uptake of liposomes and/or entrapped markers from jejunum as a function of time. Exactly $2.0 \mathrm{ml}$ of liposomal dispersion (containing about $24 \mu \mathrm{mol}$ of phospholipid) was introduced into the lumen of the segment. The contents were oscillated back and forth twice before taking the "zero time" sample. The fluid was pumped at $0.075 \mathrm{ml} / \mathrm{s}$ and the direction was reversed approximately every 10 $s$ by the two-directional piston pump. A $25-\mu 1$ sample was taken every $5 \mathrm{~min}$ for $30 \mathrm{~min}$ for assay.

Since the kinetic data obtained by this procedure were too scattered to ascertain whether or not entrapped markers or liposomes are transported through the jejunum (see Results), efforts were concentrated in an attempt to determine the total uptake of liposomes and/or entrapped markers from the lumen at the conclusion of the 3-h experiment. Exactly $2 \mathrm{ml}$ test preparation was introduced into the lumen of the intestinal segment and the contents were oscillated back and forth twice and then maintained under static condition. At the end of $3 \mathrm{~h}, 100 \mu 1$ of $1 \%$ Triton solution together with $2 \mathrm{ml}$ phosphate buffer were added to the lumen to facilitate disruption of the liposomes and release of their entrapped marker. This washing procedure was repeated $2-3$ times and the total washings were collected and analyzed for radioactivity. The intestinal segment was then removed, everted and thoroughly rinsed with buffer to further facilitate removal of liposomes which may have adhered to the intestinal wall. The volume of each washing was measured and an aliquot was taken and counted for radioactivity. The degrees of recovery of both liposomal lipid and marker were determined by dividing the total amount recovered from the washing solutions by the amount originally administered to the rat lumen.

\section{Results}

Effect of acidity, bile salt and lipase on stability of liposomes

Rowland and Woodley (1980) reported that in the presence of $10 \mathrm{mM}$ bile salts, more than $80 \%$ of the entrapped ${ }^{125} \mathrm{I}$-polyvinylpyrrolidone (PVP) was released within $30 \mathrm{~min}$ from liposomes of varying lipid composition. Only $\mathbf{L}-\alpha$-phosphatidylcholine distearoyl/cholesterol liposomes showed reasonable stability, releasing $14 \%$ of the entrapped marker within $30 \mathrm{~min}$. In agreement with Rowland and Woodley, when egg lecithin/ cholesterol liposomes containing entrapped carboxyfluorescein were incubated with $10 \mathrm{mM}$ taurocholic acid, more than $95 \%$ of the dye was released from the liposomes within $5 \mathrm{~min}$. Therefore, efforts were concentrated on determining the effects of $\mathrm{pH}$, bile salt and lipase on stability of

TABLE 1

Percentage of entrapped markers released from $\mathrm{L}-\alpha$-phosphatidylcholine distearoyl/cholesterol liposomes under various conditions

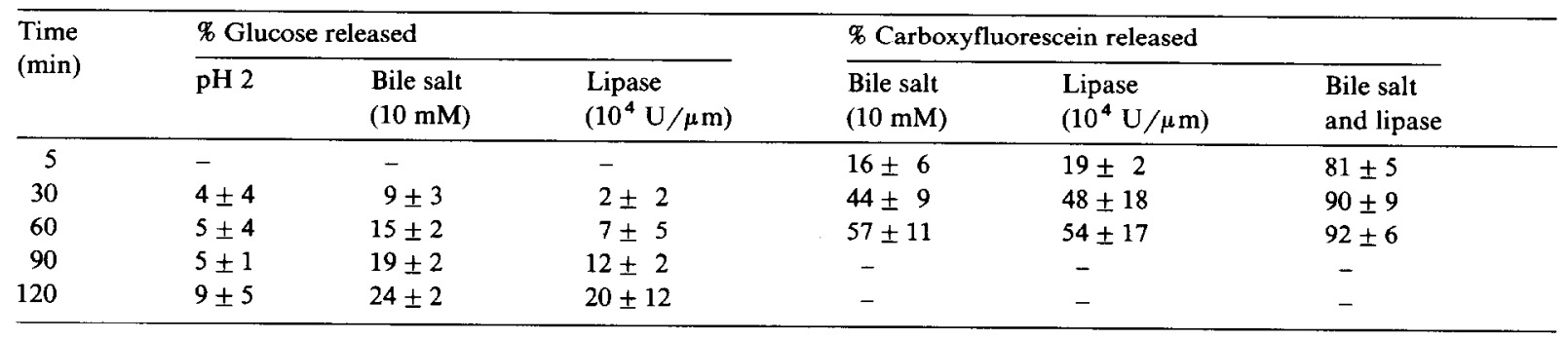


L- $\alpha$-phosphatidylcholine distearoyl/cholesterol liposomes. The results of these studies are shown in Table 1.

\section{In vitro uptake studies}

\section{Diffusion cell experiments}

Representative permeation plots for methanol, ethanol, butanol, progesterone and hydrocortisone using full-thickness jejunal membranes, are shown in Fig. 1. Whereas no apparent lag time was observed for the free alkanols, progesterone and hydrocortisone show lag times of about 30-40 min. Most importantly, neither the liposomes (as determined by their labelled lipids) nor their entrapped markers (hydrocortisone and progesterone) were detected in the receiver compartment at the end of the experiment (120 min).

\section{Intestinal everted-sac experiments}

Neither free nor entrapped PEG-4000 (a nonabsorbable marker) was detected in the everted sacs at the end of the 2-h experiment. Whereas a significant percentage of the free glucose (an absorbable marker) can penetrate into the everted sacs within $2 \mathrm{~h}(7.7 \pm 1.8 \%)$, lipsomally entrapped glucose was not detected in the everted sacs dur- ing the same time period. Essentially all of the free PEG-4000, entrapped PEG-4000 and entrapped glucose was recovered from the incubation media at the end of $2 \mathrm{~h}(>97.5 \%)$ whereas only $88 \%$ of the free glucose was recovered during the same time period. Therefore, liposomal entrapment does not facilitate the transport of a non-absorbable marker, and more importantly, retards the transport of a water-soluble absorbable marker as determined with the everted sac system.

\section{In situ uptake studies}

When determining the liposomal uptake using the modified Doluisio technique, flocculation or clumping of the liposomes was visually observed within $1 \mathrm{~min}$ after their introduction into the lumen. This aggregation, leading to further heterogeneity of the dispersion and inaccessibility of the liposomes to sampling, resulted in highly scattered data (Fig. 2). Attempts to reduce the degree of liposomal flocculation by loosening and removing mucus by pretreatment of the intestinal segments with chymotrypsin solution $(10 \mathrm{mg} / \mathrm{ml})$ were unsuccessful. The uptake of free $\left[{ }^{14} \mathrm{C}\right]$ butanol dissolved in PBS in the presence of $\mathbf{L}-\alpha$-phosphatidylcholine distearoyl/cholesterol liposomes followed first-order kinetics with an uptake rate con-
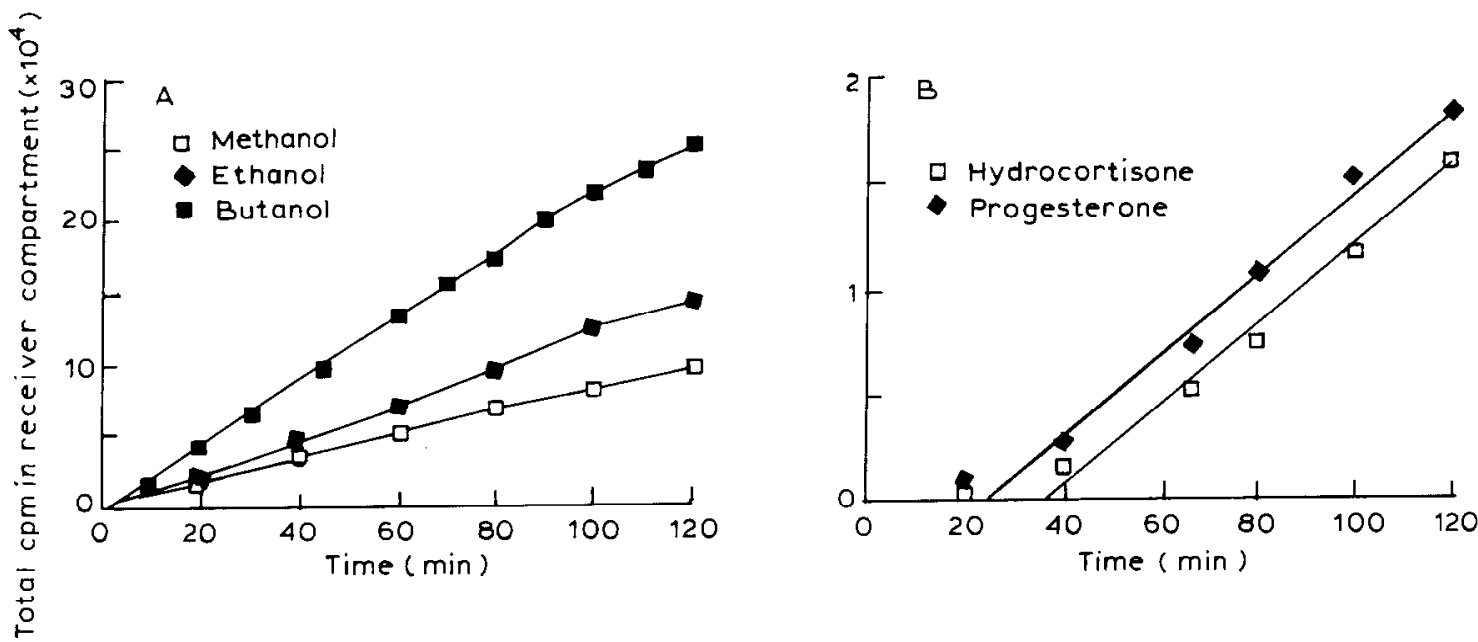

Fig. 1. Representative permeation plots of (a) alkanols and (b) steroids through full-thickness intestinal membranes of rats as determined by diffusion cell studies. The receiver compartment was filled with $1.8 \mathrm{ml}$ buffer and the donor compartment was filled with $1.8 \mathrm{ml}$ of a buffered solution of free permeant (equilibrated to $37^{\circ} \mathrm{C}$ ). Samples were taken from the donor and receiver compartments at various time intervals for $2 \mathrm{~h}$ and the samples were assayed for radioactivity. See text for experimental details. 


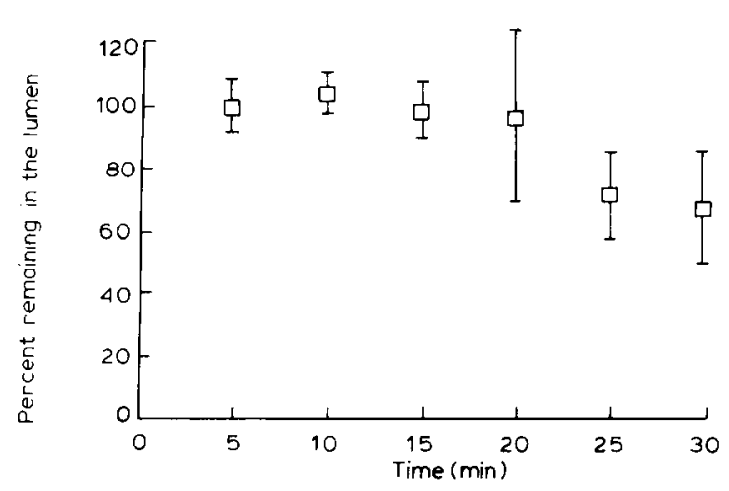

Fig. 2. Representative uptake plot of $L-\alpha$-phosphatidylcholine distearoyl/cholesterol multilamellar vesicles from the jejunum of the rat as determined by the modified Doluisio method (bar denotes S.D.). Exactly $2.0 \mathrm{ml}$ of liposomal dispersion (containing about $24 \mu \mathrm{mol}$ of phospholipid) were introduced into the lumen of the segment. The contents were oscillated back and forth twice before taking the "zero time" sample. The fluid was pumped at $0.075 \mathrm{ml} / \mathrm{s}$ and the direction was reversed approximately every $10 \mathrm{~s}$ by the two-directional piston pump. A $25 \mu 1$ sample was taken every $5 \mathrm{~min}$ for $30 \mathrm{~min}$ for assay. See text for experimental details.

stant of $1.59 \times 10^{-3} \mathrm{~s}^{-1}$. This value was the same in the absence of liposomes indicating that liposomes, even in their aggregated state, do not affect the rate of uptake of a dissolved marker.

Examination of the in vitro data (from both diffusion cell and everted-sac experiments) indicates the absence of uptake of liposomes or entrapped markers. Furthermore, the kinetic data obtained from the modificd Doluisio technique are too scattered to conclude whether or not entrapped markers or liposomes are transported through the gastrointestinal tract. Therefore, mass balance studies using the Doluisio method were performed to ascertain the degree of recovery of liposomes and/or their entrapped markers $3 \mathrm{~h}$ after administration of the dispersion and to determine whether the liposomes were adsorbed onto or absorbed and trapped within the intestinal membranes. The recoveries of liposomes and entrapped markers from the jejunal segment after 3 $\mathrm{h}$ are shown in Table 2.

When the washing procedure was performed in the absence of the Triton X-100, the total recovery of radiolabelled phospholipids ranged from 72 to $98 \%$ with an average recovery of $84 \pm 11 \%$. The detergent is needed to disrupt the liposomes since their aggregation appears to result in physical retainment in the dead spaces of the S-shaped gastrointestinal segment. It is noteworthy that by employing this revised washing procedure, more than $90 \%$ of both liposomal lipids and their entrapped markers were recovered in all experiments.

Schwinke et al. (1984) were the first group to apply the Doluisio technique to explore factors which might influence the uptake of liposomes from the rat jejunum, and to determine the fate of liposomes and their entrapped markers. Although they reported "apparent" uptake of liposomes from the jejunum, entrapped markers failed to reach the systemic circulation as determined by sampling of expected target tissues, i.e., lymph nodes, liver and spleen. About $30 \%$ of the liposomes (and their markers) were recovered from jejunal tissue upon analysis by tissuc oxidation. The greater recovery from intestinal washings of lipid label and entrapped marker in the present study ( $>90 \%$ vs $70 \%$ ) can be attributed to the use of detergent in the washing procedure.

TABLE 2

Total recovery of liposomes and entrapped markers from the rat jejunum at 3 has determined by the in situ Doluisio technique

\begin{tabular}{|c|c|c|c|}
\hline \multirow[t]{2}{*}{ Liposome composition } & \multirow[t]{2}{*}{ Entrapped marker } & \multicolumn{2}{|c|}{ Percentage recovery } \\
\hline & & Lipid & Marker \\
\hline$\left[{ }^{14} \mathrm{C}\right]$ distearoyl phosphatidylcholine:cholesterol & - & $93 \pm 6$ & - \\
\hline$\left[{ }^{14} \mathrm{C}\right]$ distearoyl phosphatidylcholine:cholesterol & {$\left[{ }^{3} \mathrm{H}\right] \mathrm{PEG}$} & $92 \pm 7$ & $90 \pm 7$ \\
\hline distearoyl phosphatidylcholine: $\left[{ }^{3} \mathrm{H}\right]$ cholesterol & {$\left[{ }^{14} \mathrm{C}\right]$ sucrose } & $92 \pm 5^{-}$ & $97 \pm 4$ \\
\hline
\end{tabular}




\section{Discussion}

The liposomes were fairly stable at $\mathrm{pH} \mathrm{2,} \mathrm{in}$ agreement with the data of Rowland and Woodley (1980). Patel and Ryman (1977) also showed that up to $75 \%$ of the insulin entrapped in egg lecithin/cholesterol/diacetyl phosphate liposomes was protected during passage through the stomach after oral administration to rats. Thus, acid conditions may not pose a major threat even to liposomes having compositions that generally tend to make them leaky (unsaturated phospholipids and charged head groups).

After incubation of the $L-\alpha$-phosphatidylcholine distearoyl/cholesterol liposomes with $10 \mathrm{mM}$ taurocholic acid, $9 \%$ of the entrapped glucose was released within the first $30 \mathrm{~min}$ and $15 \%$ of the marker was released at the end of $60 \mathrm{~min}$. The liposomes appeared to be much more leaky when carboxyfluorescein was used as a marker under the same conditions (44\% release in $30 \mathrm{~min}$ and $57 \%$ release in $60 \mathrm{~min}$ ). Rowland and Woodley (1980) reported that 14 and $16 \%$ of the entrapped markers ( ${ }^{125} \mathrm{I}$-PVP) were released from $\mathrm{L}-\alpha$-phosphatidylcholine distearoyl/cholesterol liposomes in the presence of $10 \mathrm{mM}$ bile salts in 30 and 60 min, respectively. Thus, the apparent stability of liposomes is dependent on the marker employed.

Rowland and Woodley (1980) investigated the effects of $\mathrm{pH}$, bile salts and pancreatic lipase on the stability of liposomes having lipid compositions representative of those used in in vivo oral absorption studies. Most of these liposomes remained intact at low $\mathrm{pH}$ values while almost all disintegrated upon exposure to $10 \mathrm{mM}$ bile salts. In most cases, bile led to the release of over $80 \%$ of an entrapped ${ }^{125}$ I-PVP marker within $30 \mathrm{~min}$. L- $\alpha$-Phosphatidylcholine distearoyl/cholesterol liposomes showed the best stability, as only $14 \%$ of the marker was released within $30 \mathrm{~min}$. L- $\alpha$ Phosphatidylcholine distearoyl/cholesterol liposomes also proved to be reasonably resistant to hydrolysis upon incubation with phospholipid lipase, since only $10 \%$ marker leakage was reported at $37^{\circ} \mathrm{C}$ after $1 \mathrm{~h}$ of incubation. Richards and Gardner (1978) reported that small unilamellar vesicles prepared from egg lecithin, dipalmitoyl phosphatidylcholine or dipalmitoyl phosphatidyl- ethanolamine were disrupted in the presence of taurocholate and glycocholate. Liposomes composed of phosphatidylcholine : cholesterol : stearic acid $(7: 2: 2.5)$ released almost $100 \%$ of their entrapped insulin when incubated for $3 \mathrm{~h}$ with a 2 or 4-fold dilution of rat bile which was obtained by cannulation (Hashimoto and Kawada, 1979). The effect of bile salts on the stability of liposomes seems to be dependent on their lipid composition. It has been suggested that "solid" liposomes, i.e., liposomes whose phospholipids are in the gel state, are most resistant to bile salt disruption than "fluid" liposomes, i.e. liposomes whose phospholipids are in the liquid crystalline state (Richards and Gardner, 1978).

Whereas lipase caused no significant leakage of entrapped glucose for the first $30 \mathrm{~min}$, incubation for 60 and $120 \mathrm{~min}$ resulted in $10 \%$ and $20 \%$ release of the entrapped marker, respectively. Again, the liposomes appeared to be much more leaky when carboxyfluorescein was used as a marker under the same conditions ( $48 \%$ release in $30 \mathrm{~min}$ and $54 \%$ release in $60 \mathrm{~min}$ ). Rowland and Woodley (1980) reported $10 \%$ leakage of entrapped marker after $60 \mathrm{~min}$ incubation with lipase. Other studies have also shown that phospholipids of liposomes are susceptible to attack by phospholipases present in the digestive lumen (Paltauf, 1969, Scow et al., 1967). Menashe et al. (1986) and Lichtenberg et al. (1986) reported that small unilamellar vesicles prepared from dipalmitoyl phosphatidylcholine were significantly lysed on incubation with phospholipase whereas lower leakage rates were observed for large unilamellar vesicles of the same composition. The reason given for the difference in stability is the tighter packing of the lipid in the larger vesicles.

Loss of entrapped carboxyfluorescein in the presence of both bile and lipase (more than $80 \%$ release within $5 \mathrm{~min}$ ) demonstrates a significant synergistic effect of these two destabilizing agents. This effect is not observed at later times (30 and $60 \mathrm{~min}$ ) since disruption is essentially complete within $30 \mathrm{~min}$. Hunt and Jones (1984) also demonstrated a significant synergism in the combined effects of bile salts and phospholipase with respect to the leakage of $\mathrm{Dy}^{3+}$ from egg lecithin small unilamellar vesicles. 
Deshmukh et al. (1981) suggested that the only function of liposomes as an oral dosage form is to protect the entrapped drug in the gastrointestinal tract and thereby increase the uptake of drugs after oral administration. However, there are other reports (Rowland and Woodley, 1980) which suggest that little to no protection is actually realized when liposomes composed of unsaturated phospholipids and/or charged lipids are exposed to the environment of the gastrointestinal tract. This suggestion is supported by a number of studies (Wu et al., 1982; Mauk and Gamble, 1979; Hwang, 1983) on liposome disruption in various tissues of the living mouse as investigated by $\gamma$-ray perturbed angular correlation spectroscopy. For example, Mauk and Gamble (1979) report that L- $\alpha$ phosphatidylcholine distearoyl/cholesterol unilamellar vesicles are destroyed within a few minutes after oral administration. Thus, the almost complete loss of liposomally entrapped carboxyfluorescein in the presence of both bile and lipase indicates that even $\mathrm{L}-\alpha$-phosphatidylcholine distearoyl/cholesterol liposomes may not be able to carry out their most elementary function, that of offering protection to their encapsulated drug in the harsh environment in the gastrointestinal tract.

The second suggested rationale for the use of liposomes as an oral drug delivery system is to increase the absorption of poorly absorbed drugs from the gastrointestinal tract. The results of this study indicate that this goal may also be unattainable. For each of the 3 intestinal uptake models used (diffusion cells, everted sacs, and the modified Doluisio technique) there was a complete absence of transport of all compounds tested when these markers were entrapped in liposomes indicating that liposomes cannot be transported across the membrane, and liposomal entrapment of drug fails to facilitate transport of non-absorbable drugs and prevents the transport of $a b-$ sorbable ones.

Other studies using the in vitro everted sac technique support this view. Whitmore and Wheeler (1979) suggested that neither intact liposomes nor their entrapped solutes were absorbed from the muscosal solution and the recovery from the mucosal solution was $99 \pm 1 \%$. Seiden and
Lichtenberg (1979) also concluded that only a negligible fraction of radiolabelled egg lecithin/ cholesterol liposomes passed through gut sacs of diabetic rats within $1 \mathrm{~h}$. However, a number of studies seem to support the opposing view that liposomal entrapment increases the gastrointestinal transport of poorly absorbed drugs.

Rowland and Woodley (1981a and b), using the in vitro everted sac technique, suggested that gut epithelial cells take up intact liposomes by adsorptive endocytosis. Their conclusion was based on the observation of a 4-fold increase in tissue uptake and a 2-fold increase in appearance of marker on the serosal side of liposomally entrapped ${ }^{125} \mathrm{I}$ PVP over that of the free PVP. However, the increase in tissue uptake is not necessarily reflective of absorptive cell uptake since liposomes may adhere to the outer layer of the intestinal membrane and the increase in uptake may reflect adsorption rather than absorption. Furthermore, when the amount of marker transported into the serosal space is calculated from their data, the total percentage of either liposomal or free PVP transported across the everted sacs is less than $1 \%$. Thus, their results seem to be in agreement with other groups (Whitmore and Wheeler, 1979) which show no significant transport or liposomes or their entrapped markers across intestinal membranes.

Kimura et al. (1984), also using everted sacs, reported that intestinal epithelial cells slowly take up liposomes by endocytosis and the mechanism is dependent on liposomal lipid composition. They suggested that egg lecithin liposomes are endocytosed by epithelial cells while L- $\alpha$-phosphatidylcholine distearoyl liposomes are not, since the transport of fluoresceinisothiocyanate-conjugated dextran (FITC-D) in egg lecithin liposomes was greater than when the marker was entrapped in L- $\alpha$-phosphatidylcholine distearoyl liposomes but similar to that of the transport of the free marker. These results contradict in vitro studies which demonstrate that "solid" liposomes are more likely to undergo endocytosis than are the "fluid" liposomes (Rowland and Woodley, 1981a; Papahadjopoulos et al., 1975). They further reported that the clearance of liposomally entrapped FITC$\mathrm{D}$ was significantly reduced when metabolic inhibitors were introduced into the system. How- 
ever, since the total percentage transported in either case was less than $0.1 \%$, the results may have little practical importance. Furthermore, they reported that egg lecitin liposomes were more stable than L- $\alpha$-phosphatidylcholine distearoyl liposomes upon incubation with everted intestine, a result which contradicts previous reports (Rowland and Woodley, 1980; Richards and Gardner, 1978).

Patel et al. (1985) used the isolated perfused intestinal loop system (Shichir et al., 1973; Sakai et al., 1980) to study the intestinal absorption of liposomally entrapped macromolecules. The transport of liposomes and their entrapped macromolecules were monitored by measuring the presence of the markers, cholesterol, insulin and PVP in the venous effluent. Their results showed that although intact liposomes were not transported across the intestinal membrane, liposomally entrapped macromolecules did appear in the venous effluent. They concluded that liposomes are taken up by epithelial cells and then degraded intracellularly, releasing the markers through the basement membrane into the venous effluent. However, in their experiments, aqueous solutions of the non-absorbable markers were transported rapidly through the intestinal membrane into the venous effluent suggesting that the intestinal membranes had already lost their barrier properties during the preparative stage. Furthermore, since they reported that $85 \%$ of the L- $\alpha$-phosphatidylcholine distcaroyl liposomes were degraded at the end of the perfusion, it is not reasonable to draw the conclusion that intact liposomes are taken up by epithelial cells.

The combined evidence from the in vitro diffusion cell and everted sac experiments suggest that intact liposomes are not taken up by epithelial cells and that liposomes do not facilitate the uptake of entrapped markers. In situ studies seem to confirm this viewpoint since almost all of the liposomes and their entrapped markers remain in the intestine even after $3 \mathrm{~h}$.

\section{References}

Arrieta-Molero, J.F., Aleck, K., Sinha, M.K., Brownscheidle, C.M., Shapiro, L.J. and Sperling, M.A., Oral administered liposome-entrapped insulin in diabetic animals. A critical assessement. Horm. Res., 16 (1982) 249-256.

Bangham, A.D., Standish, M.M. and Watkins, J.C., Diffusion of univalent ions across the lamellae of swollen phospholipids. J. Mol. Biol., 13 (1965) 238-252.

Deshmukh, D.S., Bear, W.D. and Brockerhoff, H., Can intact liposomes be absorbed in the gut? Life Sci., 28 (1981) 239-242.

Hashimoto, A. and Kawada, J., Effects of oral administration of positively charged insulin liposomes on alloxan diabetic rats: preliminary study. Endocrinol. Jap., 26 (1979) 337-344.

Hemker, H.C., Hermens, W.T., Muller, A.D. and Zwaal, R.F., Oral treatment of haemophilia $A$ by gastrointestinal absorption of factor VIII entrapped in liposomes. Lancet, 1 (1980) 70-71.

I Iunt, G.R.A. and Jones, J.C., Application of ${ }^{1}$ H-N.M.R. to the design of liposomes for oral use. Synergistic activity of bile salts and pancreatic phospholipase $A_{2}$ in the induced permeability of small unilamellar phospholipid vesicles. $J$. Microencapsulation, 1 (1984) 113-122.

Huq, A.S., Ho, N.F.H., Husari, N., Flynn, G.L., Jetzer, W.E. and Condie Jr., L., Permeation of water contaminative phenols through hairless mouse skin. Arch. Environ. Contam. Toxicol., 15 (1986) 557-566.

Hwang, K.J., The use of gamma ray perturbed angular correlation technique for the study of liposomal integrity in vitro and in vivo. In Liposome Technology, Ch. 16, CRC, Boca Raton, FL, 1983.

Kawada, J., Tanaka, N. and Nozaki, Y., No reduction of blood glucose in diabetic rats after oral administration of insulin liposomes prepared under acidic conditions. Endocrinol. Jpn., 28 (1981) 235-238.

Kimura, T., Higaki, K. and Sezaki, H., Transmucosal passage of liposomally-entrapped drugs in rat small intestine. Pharm. Res. (1984) 221-224.

Lichtenberg, D., Romero, G., Menashe, M. and Biltonen, R.L., Hydrolysis of dipalmitoylphosphatidylcholine large unilammelar vesicles by porcine pancreatic phospholipase $\mathrm{A}_{2} . J$. Biol. Chem., 261 (1986) 5334-5340.

Mauk, M.R. and Gamble, R.C., Stability of lipid vesicles in tissues of the mouse: a $\gamma$-ray perturbed angular correlation study. Proc. Natl. Acad. Sci. U.S.A., 76 (1979) 765-769.

Menashe, M., Romero, G., Biltonen, R.L. and Lichtenberg, D., Hydrolysis of dipalmitoylphosphatidylcholine small unilamellar vesicles by porcine pancreatic phospholipase $\mathrm{A}_{2}$. J. Biol. Chem., 261 (1986) 5328-5333.

Nagata, M., Yotsuyanagi, T., Nonomura, M. and Ikeda, K., Coagulation recovery after warfarin-induced hypoprothrombinaemia by oral administration of liposomally-associated vitamin $\mathrm{K}_{1}$ to rabbits. J. Pharm. Pharmacol, 36 (1984) 527-533.

Paltauf, F., The intestinal absorption of 1,2- and 1,3-dialkyl glycerol ethers and of diether phospholipids. Biochim. Biophys. Acta, 176 (1969) 818-827.

Papahadjopoulos, D., Poste, G. and Mayhew, E., The interaction of phospholipid vesicles with mammalian cells in vitro. Biochem. Soc. Trans., 3 (1975) 606-608.

Patel, H.M., Tuzel, N.S. and Stevenson, R.W., Intracellular 
digestion of saturated and unsaturated phospholipid liposomes by muscosal cells. Possible mechanism of transport of liposomally entrapped macromolecules across the isolated vascularly perfused rabbit ileum. Biochim. Biophys. Actu, 839 (1985) 40-49.

Patel, H.M. and Ryman, B.E., Orally administered liposomally entrapped insulin. Biochem. Soc. Trans., 5 (1977) 1739-1741.

Richards, M.H. and Gardner, C.R., Effects of bile salts on the structural integrity of liposomes. Biochim. Biophys. Acta, 543 (1978) 508-522.

Rowland, R.N. and Woodley, J.F., The stability of liposomes in vitro to $\mathrm{pH}$, bile salts and pancreatic lipase. Biochim. Biophys. Acta, 620 (1980) 400-409.

Rowland, R.N. and Woodley, J.F., The uptake of distearoylphosphatidylcholine/cholesterol liposomes by rat intestinal sacs in vitro. Biochim. Biophys. Acta, 673 (1981a) 217-223.

Rowland, R.N. and Woodley, J.F., Uptake of free and liposome-entrapped ${ }^{125}$ I-labelled PVP by rat intestinal sacs in vitro: evidence for endocytosis, Biosci. Rep., 1 (1981b) 399-406.

Sakai, K., Akima, M., Hinolara, Y., Sasaki, M. and Niki, R., Vascularly perfused rat small intestine: a research model for drug absorption. Jpn. J. Pharmacol., 30 (1980) 231-241.

Schwinke, D.L., Ganesan, M.G. and Weiner, N., In situ jejunal uptake from liposomal systems. Int. J. Pharm., 20 (1984) 119-127.

Scow, M.O., Stein, Y. and Stein, O., Incorporation of dietary lecithin and lysolecithin into lymph chylomicrons in the rat. J. Biol. Chem., 242 (1967) 4919-4924.

Seiden, A. and Lichtenberg, D., Transport of liposome components in rat everted intestinal loops. J. Pharm. Pharmacol, 31 (1979) 414-415.
Sessa, G. and Weissmann, G., Incorporation of lysozyme into liposomes, J. Biol. Chem., 13 (1970) 3295-3301.

Shichir, M., Etani, N., Kawamuri, R., Karasaki, K., Okada, A., Shigeta, Y. and Abe, H., Absorption of insulin from perfused rabbit small intestine in vitro. Diabetes, 22 (1973) 459-465.

Sila, M., Au, S. and Weiner, N., Effects of Triton X-100 concentration and incubation temperature on carboxyfluorescein release from multilamellar liposomes. Biochim. Biophys. Acta, 859 (1986) 165-170.

Tragl, K.H., Pohl, A. and Kinast, H., Oral administration of insulin by means of liposomes in animal experiments. Wien. Klin. Wochenschr, 91 (1979) 448-451.

Ueno, M., Nakasaki, T., Hirikoshi, I. and Sakuragawa, N., Oral administration of liposomally-entrapped heparin to beagle dogs. Chem. Pharm. Bull., 30 (1982) 2245-2247.

Whitmore, D.A. and Wheeler, K.P., The fate of liposomes in the rat small intestine. Biochem. Soc. Trans., 7 (1979) 929-931.

Wilson, T.M. and Wiseman, G., Use of sacs of everted small intestine for study of transference of substance from mucosal to serosal surface. J. Physiol. (London), 123 (1954) 116-125.

Wu, P.-S., Wu, H.-M., Tin, G.W., Schuh, J.R., Croasmun, W.R., Baldeschwieler, J.D., Shen, T.Y. and Ponpipom, M.M., Stability of carbohydrate-modified vesicles in vivo: comparative effects of ceramide and cholesterol glycoconjugates. Proc. Natl. Acad. Sci. U.S.A., 79 (1982) 5490-5493.

Young, S.P., Baker, E. and Huehns, E.R., Liposome entrapped desferrioxamine and iron transporting ionophores: a new approach to iron chelating therapy. Br. J. Haematol., 41 (1979) 357-363 